\title{
Research into the influence of isocyanate resin compatibilizer on ABS/PET alloys' mechanical properties
}

\author{
Bo-Fen Huang ${ }^{1, a}$, Xiang Liang ${ }^{2, b^{*}}$, Xiao Li ${ }^{3, c}$, Dan Li ${ }^{4, d}$ \\ ${ }^{1}$ School of Materials Science and Engineering, Nanchang University, Nanchang, China \\ $2^{*}$ Jiangxi University of Traditional Chinese Medicine, Nanchang, China \\ ${ }^{3}$ Hefei Genius Advanced Material Co.,Ltd, Hefei, China \\ ${ }^{4}$ National engineering technology research center for LED on Si substrate,Nanchang, China \\ ahuangbf90@126.com, lliangx90@126.com, 'jasonlixiao@163.com \\ didan6570429@sina.com
}

Keywords: PET; ABS; isocyanate resin (ITTR); mechanical properties; moleculestructure

Abstract. The ABS/PET and ITTR/ABS/PET alloys were prepared by melt blending method using hard-shell structure of isocyanate terminated silicone resin as compatibilizer. Moleculestructure of ITTR, PET and the alloys had been characterized by FTIR and mechanical properties such as impact strength, bending strength had also been measured. The best alloy recipe was obtained in the paper. FTIR show ITTR resin in isocyanate groups reacted to hydroxyl groups in PET; the isocyanate group disappeared, reducing the number of hydroxyl groups; ITTR between PET and ABS resin played the role of "molecular bridge". When quality ratio of the ITTR/ABS/PET alloy equals $4 / 30 / 70$, the alloy modification effect for the mechanical properties is optimized. At this point the tensile strength, bending strength and impact strength of the alloy increases significantly to $59.97 \mathrm{MPa}, 113.79 \mathrm{MPa}$ and $9.61 \mathrm{KJ} / \mathrm{m}^{2}$ respectively, exceeding pure PET by $34.74 \%$, $36.19 \%$ and $66.84 \%$ respectively.

\section{Introduction}

Polyethylene terephthalate (PET), which belongs to crystalline saturated polyester, has the advantages such as good mechanical properties, hardness, rigidity, small absorption rate under wet conditions. While it also exists the shortcomings like poor shock intensity, difficulty of molding processing, which these limit its application [1,2].

ABS resin is composed of three monomers butadiene, styrene and acrylonitrile copolymer composed. It owns advantages such as good toughness and hardness, phase equalizationwhen is used as PET resin modifier [3,4]. However, ABS resin is an amorphous resin because PET is a crystalline resin, resulting in poor compatibility of PET and ABS phase when they are blended as an alloy.

ITTR resin, shown as pale yellow transparent liquid, is a Isocyanate resin with "hard shell" structure. ITTR molecules, containing highly reactive isocyanate groups and siloxane groups, is stable at low temperatures and effectively release active isocyanate group at a high temperature. The resin has excellent flame retardancy, weather resistance and thermal stability. It has a good combination of a variety of resin materials. Adding ITTR as a compatibilizer can form powerful bond between the various components of the blend of materials, so that each component compatibility is improved, thereby improving alloy performance.

In this paper, ITTR resin is as a compatibilizer in order to explore the impact on the ABS / PET alloy mechanical properties and microstructure. 


\section{Experimental}

\section{Materials}

PET: CZ328, Taiwan Changchun Chemical Co; ABS: 749S, Zhenjiang Chi Mei Chemical Co., Ltd.; isocyanate resin (ITTR) : China National Pharmaceutical Group.

\section{Equipment and instruments}

Twin-screw extruder: SHJ-20B, Nanjing Jie Ente Electrical Company; injection molding machine: PS610BM, Shenzhen Bao Gitzo technology companies; Fourier Transform Infrared Spectrometer: FTIR-1700, US PE firm; Charpy impact test machine: XJJD-5, Chengde Jin Jian Detection Instruments; electronic testing machine: CTM420, Shenzhen Sans Materials Testing Company.

\section{Preparation of alloys}

The ABS and PET were respectively baked in an oven with 12 hours, at $80{ }^{\circ} \mathrm{C}$ and $85{ }^{\circ} \mathrm{C}$, then will be ABS, PET and ITTR were extruded in an extruder according to mixing ratio, the period of the five stages of heating temperature were respectively, $245{ }^{\circ} \mathrm{C}, 250{ }^{\circ} \mathrm{C}, 255^{\circ} \mathrm{C}, 260{ }^{\circ} \mathrm{C}, 265^{\circ} \mathrm{C}$. Extruding the blend particles after drying at $85{ }^{\circ} \mathrm{C}, 12 \mathrm{~h}$, and then injecting molding machine by injection molding.

\section{Performance Testing and Characterization}

Fourier transform infrared spectroscopy (FTIR): scraping a small amount of powder samples with $\mathrm{KBr}$ tablet, scanning in a Fourier transform infrared spectrometer, the scanning frequency was 32 times with scan interval of wavenumber $2 \mathrm{~cm}^{-1}$.Mechanical properties: Bending strength was carried according to GB / T 9341-2008 Test, test speed was $2 \mathrm{~mm} / \mathrm{min}$; tensile strength was tested according to GB / T 1040-2006 Test, test speed was $2 \mathrm{~mm} / \mathrm{min}$; the impact strength was done according to GB / T 1843-2008 Test .

\section{Results and discussion}

\section{FTIR analysis}

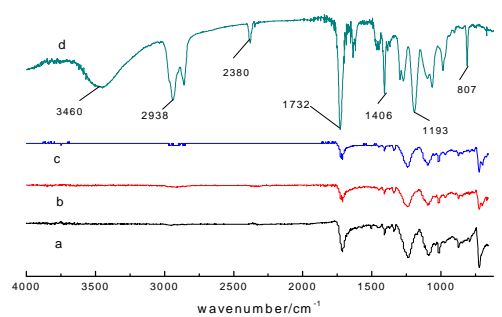

Fig.1 FT-IR

a: PET; b: ABS/PET; c: ITTR/ABS/PET; d: ITTR

Fig. 1 illustrated that curve $d$ is the infrared spectrum of ITTR resin. The figure showed that there was a broad absorption peak at about $3460 \mathrm{~cm}^{-1}$, which was $-\mathrm{OH}$ bond; there was a strong absorption peak description at about $1732 \mathrm{~cm}^{-1}$ containing a double bond $-\mathrm{C}=\mathrm{O}$, there were both absorption peaks at $2380 \mathrm{~cm}^{-1}$ and $1636 \mathrm{~cm}^{-1}$, which could be inferred the existence of cumulative double bond, comprehensive analysis showed, ITTR resin contained a $-\mathrm{N}=\mathrm{C}=\mathrm{O}$ bond; absorption peak at $1193 \mathrm{~cm}^{-1}$ was a characteristic peak of CO; $1000 \sim 1300 \mathrm{~cm}^{-1}$ had several strong absorption peaks, containing the silane group; the spectrum at $807 \mathrm{~cm}^{-1}$ showed a O-H deformation vibration. Thus, it was known that ITTR resins contained $-\mathrm{OH},-\mathrm{CH}_{3},-\mathrm{N}=\mathrm{C}=\mathrm{O}$ group.

Comprehensive curve $a, b, c$ three infrared spectra analysis, after joining ABS and ITTR resin in PET, ITTR resin in isocyanate groups and PET in reacting with hydroxyl groups, isocyanate groups disappear, reducing the number of hydroxyl groups, the performance spectrum of $\mathrm{c}$ the $2380 \mathrm{~cm}^{-1}$ absorption peak disappeared absorption peak of $3460 \mathrm{~cm}^{-1}$ significantly reduced. The ITTR resin 
and ABS, PET reaction, $1732 \mathrm{~cm}^{-1}, 1406 \mathrm{~cm}^{-1}$ and other absorption greatly reduced or absent. The above results show, ITTR resin and PET, ABS reaction between PET and ABS function as "molecular bridge".

\section{ABS / PET alloy mechanical properties \\ Impact Performance}

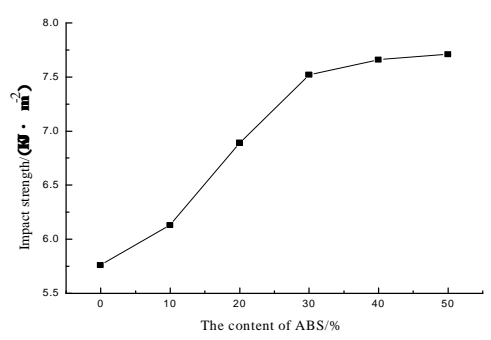

Fig. 2 Effects of different ABS content on impact strength of ABS / PET alloys

Fig. 2 showed the content of ABS on impact strength of the ABS / PET alloy. Adding ABS caused the impact strength of PET materials continued to rise. This is because the ABS contained butadiene, enabling ABS to have high impact strength. After blending with PET, when the alloy was subjected to impact, ABS could withstand some impact force and absorbed a large quantity of impact energy, thus increasing impact strength. When the ABS content was $30 \%$, the impact strength was $7.52 \mathrm{KJ} / \mathrm{m}^{2}$; when the ABS content exceeded $30 \%$, the impact strength of the alloy did not rise very obviously.

\section{Tensile Strength and Flexural Strength}

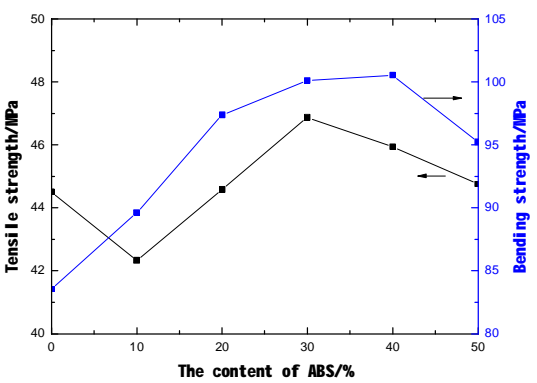

Fig. 3 Effects of different ABS content on tensile strength and bending strength of ABS / PET alloys

Fig. 3 displayed the influence of the ABS content on ABS / PET alloy's tensile and flexural properties. According to the analysis, ABS enhanced the materials' tensile properties, but the effect was not very significant. This was because even the butadiene component made ABS toughness enhancement, blending with PET made the alloy better tensile properties; however, due to a blend of ABS and PET, PET might decrease the degree of crystallinity, while its compatibility was not good. When subjected to tensile stress, cracks created between the two components, resulting in stress concentration, thus making cracks expand and causing fracture. Hence, this leaded to the enhance of tensile properties, even though there was only a slight enhance.

After the addition of the ABS, the bending strength of the blend gradually increased because the ABS itself has good bending properties. By blending with the PET, ABS can be assessed in a part of the force, thus improving material bending performance; and after addition of an excess of ABS, ABS were gathered on a PET substrate, causing increased separation between the two components. Thus, the bending strength decreased.

Based on the above experimental data, when ABS content was 30\%, the ABS/PET alloy owned best mechanical properties. Experiment determined when the ABS content was $30 \%$, selecting 
ITTR as a compatibilizer, researching the influence of ITTR on ABS / PET alloy mechanical proper ties.

\section{ITTR / ABS / PET composite mechanical properties Impact Strength}

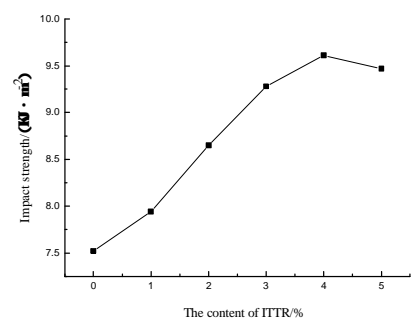

Fig. 4 The effect of ITTR content on impact strength of ABS / PET alloys

As fig. 4 illustrated, the ABS / PET alloys blended with ITTR resin, the impact resistance of the alloy was significantly increased. The reasons were ITTR, as a compatibilizer, greatly improved the ability of binding between ABS and PET. The binding force between the two phases was enhanced by the impact force, they became a common body force. Therefore, the alloy's impact performance was improved. When ITTR compatibilizer exceeded 4\%, the impact strength of the alloy was showing a downward trend. This is because too much compatibilizer leaded to blends presented a third component, destroyed the original two-phase equilibrium and resulted in performance degradation. When ITTR content was $4 \%$, the impact strength of the blend was $9.61 \mathrm{KJ} / \mathrm{m}^{2}$, increasing 66.84\% compared with PET.

\section{Tensile and Flexural Properties}

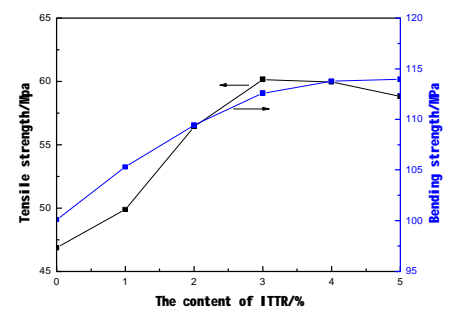

Fig. 5 ITTR influence the content of ABS / PET alloy tensile and flexural strength

The effect of ITTR content on tensile strength and bending strength of ABS / PET alloys in Fig.5. As shown, after ITTR compatibilizer was added, pulled tensile strength of ABS / PET blends showed a upward trend, this was because when the compatibilizer made the PET and ABS good two-phase connection together, compatibility greatly improved when ITTR content was $4 \%$, the tensile strength was $59.97 \mathrm{MPa}$, increasing $34.73 \%$ compared to the PET.

With the increasing amount of ITTR compatibilizer, the bending strength of the alloy kept growing, when the amount exceeded $4 \%$, the trend was levelling off. This was because the added ITTR compatibilizer alloy in ABS and PET compatibility of the two phases was improved, the good bending performance of ABS played a good role, resulting in the improve of the blends' bending properties, when the content of ITTR was $4 \%$, the bending strength of the alloy was 113.79 , increasing $36.19 \%$ compared with the PET.

\section{Conclusions}

FTIR analysis showed that ITTR resin in isocyanate groups reacted to hydroxyl groups in PET; the isocyanate group disappeared, reducing the number of hydroxyl groups; ITTR between PET and ABS resin played the role of "molecular bridge". When ITTR content reached 4\%, ITTR resin owned the best modification effect on the mechanical properties of ABS/PET, ITTR / ABS / PET alloy achieved optimum mechanical properties. Meanwhile, ITTR / ABS / PET alloy's the tensile 
strength, bending strength, impact strength significantly increased, respectively reaching 59.97MPa, 113.79MPa, $9.61 \mathrm{KJ} / \mathrm{m}^{2}$, had a $34.74 \%, 36.19 \%$ and $66.84 \%$ increase compared with PET.

\section{References}

[1] Moon S I, Jin F Z,Lee C J, Tsutsumi S.: Macromolecular Symposium. Vol. 224(2005), p.287

[2] Bofen Huang, Dan Li and Zhiyuan Li:Journal of Applied Polymer Science. Vol. 122(2011), p.586

[3] Mallakpour S,Taghavi M.: Polymer. Vol.49(2008), p.3239

[4]Toth K, Czvikovszky T, Abd-Elhamid M: Radiation Physics and Chem., Vol.69(2004), p.143 